\title{
Application of covariance adjustment to seemingly unrelated multivariate regressions
}

\author{
Lichun Wang ${ }^{1, a}$, Lawrence Pettit ${ }^{b}$ \\ ${ }^{a}$ Department of Mathematics, Beijing Jiaotong University, China; \\ ${ }^{b}$ School of Mathematical Sciences, Queen Mary University of London, UK
}

\begin{abstract}
Employing the covariance adjustment technique, we show that in the system of two seemingly unrelated multivariate regressions the estimator of regression coefficients can be expressed as a matrix power series, and conclude that the matrix series only has a unique simpler form. In the case that the covariance matrix of the system is unknown, we define a two-stage estimator for the regression coefficients which is shown to be unique and unbiased. Numerical simulations are also presented to illustrate its superiority over the ordinary least square estimator. Also, as an example we apply our results to the seemingly unrelated growth curve models.
\end{abstract}

Keywords: seemingly unrelated regressions, matrix power series, two-stage estimator

\section{Introduction}

The system of seemingly unrelated regressions (SUR) has been investigated by many authors since the pioneering works of Zellner $(1962,1963)$, which can be used to model subtle interactions among individual statistical relationships. For more details, the readers are referred to Revankar (1974), Schmidt (1977), Wang (1989), Percy (1992), Liu (2000), Liu (2002). Among these, the cases of orthogonal regressors (Zeller, 1963) and triangular SUR models (Revankar, 1974) and an SUR with unequal numbers of observations (Schmidt, 1977) are more impressive. Some examples in the econometrics literature (Srivastava and Giles, 1987) suggest that the SUR model is appropriate and useful for a wide range of applications. Further, Velu and Richards (2008) focuses on some applications of reduced-rank model in the context of SUR. Alkhamisi (2010) proposes two SUR type estimators based on combining the SUR ridge regression and the restricted least squares methods as well as evaluates their performances by means of some designated criteria. Zhou et al. (2011) also employs seemingly unrelated nonparametric regression models to fit the multivariate panel data. Shukur and Zeebari (2012) considers median regression for SUR models with the same explanatory variables and obtains an interesting feature of the generalized least absolute deviations method. However, this paper will show some interesting facts about the SUR system by employing the covariance adjustment technique. We start from the system of seemingly unrelated multivariate regressions (Gupta and Kabe, 1998), namely

$$
\left\{\begin{array}{l}
Y_{1}=X_{1} B_{1}+E_{1} \\
Y_{2}=X_{2} B_{2}+E_{2}
\end{array}\right.
$$

\footnotetext{
${ }^{1}$ Corresponding author: Department of Mathematics, Beijing Jiaotong University, No.3 Shangyuancun Haidian District, Beijing 100044, China. E-mail: lchwang@bjtu.edu.cn
}

Published 30 November 2018 / journal homepage: http://csam.or.kr

(c) 2018 The Korean Statistical Society, and Korean International Statistical Society. All rights reserved. 
where $Y_{i}(i=1,2)$ are $n \times q$ observation variables; $X_{i}(i=1,2)$ are $n \times p_{i}$ matrices with full column rank; $B_{i}(i=1,2)$ are $p_{i} \times q$ unknown regression coefficients; $E_{1}$ and $E_{2}$ are random error matrices and the row variable of $\left(E_{1}, E_{2}\right)$ follow a common unspecified multivariate distribution with mean zero and covariance matrix $V$, where $V$ is a $2 \times 2$ non-diagonal partitioned matrix and given by

$$
V=\left(\begin{array}{cc}
V_{1} & D \\
D^{T} & V_{2}
\end{array}\right)
$$

where $V_{i}$ is the variance-covariance matrix of the row variable of $E_{i}(i=1,2)$ and $D$ denotes the covariance matrix between the row variable of $E_{1}$ and the corresponding row variable of $E_{2}$. The different rows of $\left(E_{1}, E_{2}\right)$ are also assumed to be uncorrelated. The case of multivariate SUR is common in biological science. For instance, if the $i^{\text {th }}$ row of $Y_{1}$ denotes the observation vector of the weight of the $i^{\text {th }}$ rabbit at $q$ different time points and the $i^{\text {th }}$ row of $Y_{2}$ denotes the observation vector of the length of the $i^{t h}$ rabbit at the same $q$ time points, and the observation values of different rabbits are uncorrelated, then the multivariate SUR (1.1) reasonably model the interactions among the observation vectors of weight and length of $n$ rabbits.

If one neglects the correlation between $Y_{1}$ and $Y_{2}$, i.e., taking $D$ as zero, then only by the first equation of the system (1.1), one would obtain the least square estimator (LSE) for $\operatorname{Vec}\left(B_{1}\right)$ as

$$
\widehat{\operatorname{Vec}}\left(B_{1}\right)=\left(I_{q} \otimes\left(X_{1}^{T} X_{1}\right)^{-1} X_{1}^{T}\right) \operatorname{Vec}\left(Y_{1}\right)
$$

and correspondingly, the LSE of the coefficients matrix $B_{1}$ is $\hat{B}_{1}=\left(X_{1}^{T} X_{1}\right)^{-1} X_{1}^{T} Y_{1}$, where $\operatorname{Vec}(A)$ denotes the direct operator of matrix $A, \otimes$, and $I_{q}$ are the Kronecker product operator and the identity matrix of $q$ order, respectively.

However, if we denote $Y=\left(Y_{1}, Y_{2}\right), B=\left(B_{1}, B_{2}\right)$, and $E=\left(E_{1}, E_{2}\right)$, then the system (1.1) can also be represented as:

$$
\operatorname{Vec}(Y)=\left(\begin{array}{cc}
I_{q} \otimes X_{1} & 0 \\
0 & I_{q} \otimes X_{2}
\end{array}\right) \operatorname{Vec}(B)+\operatorname{Vec}(E)
$$

Hence, from (1.4), one can obtain the LSE of $\operatorname{Vec}(B)$, say $\overline{\operatorname{Vec}}(B)$, and accordingly another estimator for $\operatorname{Vec}\left(B_{1}\right)$, denoted by $\overline{\operatorname{Vec}}\left(B_{1}\right)$, can be proposed since $\overline{\operatorname{Vec}}(B)^{T}=\left(\overline{\operatorname{Vec}}\left(B_{1}\right)^{T}, \overline{\operatorname{Vec}}\left(B_{2}\right)^{T}\right)$. We think it makes sense that $\overline{\operatorname{Vec}}\left(B_{1}\right)$ and its corresponding two-stage estimator version $\overline{\operatorname{Vec}}\left(B_{1}\right)_{2 \text {-stage }}$ (in case of unknown $V$ ) should outperform $\widehat{\operatorname{Vec}}\left(B_{1}\right)(1.3)$ since they take the another equation information on $B_{1}$ into account.

The covariance adjustment technique is usually employed to obtain an optimal unbiased estimator of a vector parameter $\theta$ via linearly combining an unbiased estimator of $\theta$, say $T_{1}$, and an unbiased estimator of a zero vector, say $T_{2}$ (Rao, 1967; Baksalary, 1991).

Applying the covariance adjustment technique to the estimator $\widehat{\operatorname{Vec}}\left(B_{1}\right)$, which only uses the first equation information on $\operatorname{Vec}\left(B_{1}\right)$, we firstly use $\left(I_{q} \otimes N_{2}\right) \operatorname{Vec}\left(Y_{2}\right)$ to improve $\widehat{\operatorname{Vec}}\left(B_{1}\right)$ noting $E\left[\left(I_{q} \otimes\right.\right.$ $\left.\left.N_{2}\right) \operatorname{Vec}\left(Y_{2}\right)\right]=\left(I_{q} \otimes N_{2}\right)\left(I_{q} \otimes X_{2}\right) \operatorname{Vec}\left(B_{2}\right)=0$ and obtain $\widehat{\operatorname{Vec}}\left(B_{1}\right)^{(1)}$, secondly we again improve $\widehat{\operatorname{Vec}}\left(B_{1}\right)^{(1)}$ by $\left(I_{q} \otimes N_{1}\right) \operatorname{Vec}\left(Y_{1}\right)$ due to $E\left[\left(I_{q} \otimes N_{1}\right) \operatorname{Vec}\left(Y_{1}\right)\right]=0$ and get $\widehat{\operatorname{Vec}}\left(B_{1}\right)^{(2)}$. Repeating this 
process, we obtain the following estimator sequence $(k \geq 1)$ for $\operatorname{Vec}\left(B_{1}\right)$ :

$$
\begin{aligned}
\widehat{\operatorname{Vec}}\left(B_{1}\right)^{(2 k-1)}= & \widehat{\operatorname{Vec}}\left(B_{1}\right)^{(2 k-2)}-\operatorname{Cov}\left(\widehat{\operatorname{Vec}}\left(B_{1}\right)^{(2 k-2)},\left(I_{q} \otimes N_{2}\right) \operatorname{Vec}\left(Y_{2}\right)\right) \\
& \times\left[\operatorname{Cov}\left(\left(I_{q} \otimes N_{2}\right) \operatorname{Vec}\left(Y_{2}\right)\right)\right]^{-}\left(I_{q} \otimes N_{2}\right) \operatorname{Vec}\left(Y_{2}\right), \\
\widehat{\operatorname{Vec}}\left(B_{1}\right)^{(2 k)}= & \widehat{\operatorname{Vec}}\left(B_{1}\right)^{(2 k-1)}-\operatorname{Cov}\left(\widehat{\operatorname{Vec}}\left(B_{1}\right)^{(2 k-1)},\left(I_{q} \otimes N_{1}\right) \operatorname{Vec}\left(Y_{1}\right)\right) \\
& \times\left[\operatorname{Cov}\left(\left(I_{q} \otimes N_{1}\right) \operatorname{Vec}\left(Y_{1}\right)\right)\right]^{-}\left(I_{q} \otimes N_{1}\right) \operatorname{Vec}\left(Y_{1}\right),
\end{aligned}
$$

where $\widehat{\operatorname{Vec}}\left(B_{1}\right)^{(0)}=\widehat{\operatorname{Vec}}\left(B_{1}\right), N_{i}=I_{n}-X_{i}\left(X_{i}^{T} X_{i}\right)^{-1} X_{i}^{T}(i=1,2)$, and $A^{-}$denotes any a generalized inverse matrix of $A$.

Note that $\operatorname{Cov}\left(\operatorname{Vec}\left(Y_{i}\right), \operatorname{Vec}\left(Y_{i}\right)\right)=V_{i} \otimes I_{n}(i=1,2)$ and $\operatorname{Cov}\left(\operatorname{Vec}\left(Y_{1}\right), \operatorname{Vec}\left(Y_{2}\right)\right)=D \otimes I_{n} . \operatorname{By} \operatorname{some}$ algebra computations, we obtain that for $k \geq 1$

$$
\begin{aligned}
\widehat{\operatorname{Vec}}\left(B_{1}\right)^{(2 k-1)}= & {\left[I_{q} \otimes\left(X_{1}^{T} X_{1}\right)^{-1} X_{1}^{T}\right] \sum_{i=0}^{k-1}\left(D V_{2}^{-1} D^{T} V_{1}^{-1} \otimes N_{2} N_{1}\right)^{i} \times\left[\operatorname{Vec}\left(Y_{1}\right)-\left(D V_{2}^{-1} \otimes N_{2}\right) \operatorname{Vec}\left(Y_{2}\right)\right], } \\
\widehat{\operatorname{Vec}}\left(B_{1}\right)^{(2 k)}= & {\left[I_{q} \otimes\left(X_{1}^{T} X_{1}\right)^{-1} X_{1}^{T}\right] \sum_{i=0}^{k}\left(D V_{2}^{-1} D^{T} V_{1}^{-1} \otimes N_{2} N_{1}\right)^{i} \operatorname{Vec}\left(Y_{1}\right) } \\
& -\left[I_{q} \otimes\left(X_{1}^{T} X_{1}\right)^{-1} X_{1}^{T}\right] \sum_{i=0}^{k-1}\left(D V_{2}^{-1} D^{T} V_{1}^{-1} \otimes N_{2} N_{1}\right)^{i}\left(D V_{2}^{-1} \otimes N_{2}\right) \times \operatorname{Vec}\left(Y_{2}\right)
\end{aligned}
$$

Denote $V^{-1}=\left(\begin{array}{ll}V^{11} & V^{12} \\ V^{21} & V^{22}\end{array}\right)$ and $Q=\left(V^{11}\right)^{-1} V^{12}\left(V^{22}\right)^{-1} V^{21}$. By (1.2) and the inverse of partitioned matrix, we have

$$
\begin{aligned}
Q & =\left(V^{11}\right)^{-1} V^{12}\left(V^{22}\right)^{-1} V^{21} \\
& =\left[V_{1}-D V_{2}^{-1} D^{T}\right] \cdot\left[V_{1}-D V_{2}^{-1} D^{T}\right]^{-1} D V_{2}^{-1} \cdot\left(V_{2}-D^{T} V_{1}^{-1} D\right) \cdot V_{2}^{-1} D^{T}\left[V_{1}-D V_{2}^{-1} D^{T}\right]^{-1} \\
& =D V_{2}^{-1} D^{T}\left[\left(V_{1}-D V_{2}^{-1} D^{T}\right)^{-1}-V_{1}^{-1} D V_{2}^{-1} D^{T}\left(V_{1}-D V_{2}^{-1} D^{T}\right)^{-1}\right] \\
& =D V_{2}^{-1} D^{T} V_{1}^{-1}
\end{aligned}
$$

and $\left(V^{11}\right)^{-1} V^{12}=-D V_{2}^{-1}$. Thus we have

$$
\begin{aligned}
\overline{\operatorname{Vec}}\left(B_{1}\right)= & \widehat{\operatorname{Vec}}\left(B_{1}\right)^{(\infty)}=\lim _{k \rightarrow \infty} \widehat{\operatorname{Vec}}\left(B_{1}\right)^{(2 k-1)}=\lim _{k \rightarrow \infty} \widehat{\operatorname{Vec}}\left(B_{1}\right)^{(2 k)} \\
= & {\left[I_{q} \otimes\left(X_{1}^{T} X_{1}\right)^{-1} X_{1}^{T}\right] \sum_{i=0}^{\infty}\left(Q \otimes N_{2} N_{1}\right)^{i} \times\left[\operatorname{Vec}\left(Y_{1}\right)+\left(\left(V^{11}\right)^{-1} V^{12} \otimes N_{2}\right) \operatorname{Vec}\left(Y_{2}\right)\right] } \\
= & {\left[I_{q} \otimes\left(X_{1}^{T} X_{1}\right)^{-1} X_{1}^{T}\right]\left\{I_{q n}-Q \sum_{i=0}^{\infty}\left(Q \otimes P_{2} P_{1}\right)^{i} P_{2} N_{1}\right\} } \\
& \times\left\{\operatorname{Vec}\left(Y_{1}\right)+\left[\left(V^{11}\right)^{-1} V^{12} \otimes N_{2}\right] \operatorname{Vec}\left(Y_{2}\right)\right\},
\end{aligned}
$$

where $P_{i}=I_{n}-N_{i}=X_{i}\left(X_{i}^{T} X_{i}\right)^{-1} X_{i}^{T}$ and we use the facts that $\left(Q \otimes P_{2} P_{1}\right)^{0}=I_{q} \otimes I_{n}=I_{q n}$, $\left(V^{11}\right)^{-1}\left(Q^{T}\right)^{k} V^{11}=Q^{k}$ for $k \geq 0$ and $X_{1}^{T}\left(N_{2} N_{1}\right)^{k}=-X_{1}^{T}\left(P_{2} P_{1}\right)^{k-1} P_{2} N_{1}$ for $k \geq 1$. 
Then, we integrate the above conclusions into the following theorem, which indicates the limit of the covariance adjustment sequence and the covariance of $\overline{\operatorname{Vec}}\left(B_{1}\right)$.

Theorem 1. For the system (1.1), the limit of the covariance adjustment sequence of Vec( $\left.B_{1}\right)$ equals to $\overline{\operatorname{Vec}}\left(B_{1}\right)$, i.e., $\lim _{k \rightarrow \infty} \widehat{\operatorname{Vec}}\left(B_{1}\right)^{(k)}=\overline{\operatorname{Vec}}\left(B_{1}\right)$, and

$$
\operatorname{Cov}\left(\overline{\operatorname{Vec}}\left(B_{1}\right)\right)=V_{1} \otimes\left(X_{1}^{T} X_{1}\right)^{-1}-\left[I_{q} \otimes\left(X_{1}^{T} X_{1}\right)^{-1} X_{1}^{T}\right] \cdot G \cdot\left[I_{q} \otimes X_{1}\left(X_{1}^{T} X_{1}\right)^{-1}\right],
$$

where $G=\sum_{i=0}^{\infty}\left[Q^{i} D V_{2}^{-1} D^{T}\right] \otimes\left[\left(P_{1} P_{2} P_{1}\right)^{i}-\left(P_{1} P_{2} P_{1}\right)^{i+1}\right]$.

Proof: The first conclusion follows from the above discussion. Denote $\overline{\operatorname{Vec}}\left(B_{1}\right)=M(Q)\left\{\operatorname{Vec}\left(Y_{1}\right)+\right.$ $\left.\left[\left(V^{11}\right)^{-1} V^{12} \otimes N_{2}\right] \operatorname{Vec}\left(Y_{2}\right)\right\}$ with $M(Q)=\left[I_{q} \otimes\left(X_{1}^{T} X_{1}\right)^{-1} X_{1}^{T}\right]\left\{I_{q n}-Q \sum_{i=0}^{\infty}\left(Q \otimes P_{2} P_{1}\right)^{i} P_{2} N_{1}\right\}$, we have

$$
\begin{aligned}
& \operatorname{Cov}\left(\overline{\operatorname{Vec}}\left(B_{1}\right)\right) \\
& =M(Q)\left[V_{1} \otimes I_{n}+\left(V^{11}\right)^{-1} V^{12} V_{2} V^{21}\left(V^{11}\right)^{-1} \otimes N_{2}+D V^{21}\left(V^{11}\right)^{-1} \otimes N_{2}+\left(V^{11}\right)^{-1} V^{12} D^{T} \otimes N_{2}\right] M^{T}(Q) \\
& =M(Q)\left[V_{1} \otimes I_{n}-Q V_{1} \otimes N_{2}\right] M^{T}(Q),
\end{aligned}
$$

where we use the following fact

$$
\left(V^{11}\right)^{-1} V^{12} V_{2} V^{21}\left(V^{11}\right)^{-1}+D V^{21}\left(V^{11}\right)^{-1}+\left(V^{11}\right)^{-1} V^{12} D^{T}=-D V_{2}^{-1} D^{T}
$$

Together with the expression of $M(Q)$, we have

$$
\begin{aligned}
& \operatorname{Cov}\left(\overline{\operatorname{Vec}}\left(B_{1}\right)\right) \\
&=\left\{I_{q} \otimes\left(X_{1}^{T} X_{1}\right)^{-1} X_{1}^{T}-\sum_{i=0}^{\infty}\left[Q^{i+1} \otimes\left(X_{1}^{T} X_{1}\right)^{-1} X_{1}^{T}\left(P_{2} P_{1}\right)^{i} P_{2} N_{1}\right]\right\} \\
& \times\left\{V_{1} \otimes X_{1}\left(X_{1}^{T} X_{1}\right)^{-1}-\sum_{i=0}^{\infty}\left[V_{1}\left(Q^{T}\right)^{i+1} \otimes N_{1} P_{2}\left(P_{1} P_{2}\right)^{i} X_{1}\left(X_{1}^{T} X_{1}\right)^{-1}\right]\right\} \\
&-\left\{I_{q} \otimes\left(X_{1}^{T} X_{1}\right)^{-1} X_{1}^{T}-\sum_{i=0}^{\infty}\left[Q^{i+1} \otimes\left(X_{1}^{T} X_{1}\right)^{-1} X_{1}^{T}\left(P_{2} P_{1}\right)^{i} P_{2} N_{1}\right]\right\} \\
& \times\left\{Q V_{1} \otimes N_{2} X_{1}\left(X_{1}^{T} X_{1}\right)^{-1}-\sum_{i=0}^{\infty}\left[Q V_{1}\left(Q^{T}\right)^{i+1} \otimes N_{2} N_{1} P_{2}\left(P_{1} P_{2}\right)^{i} X_{1}\left(X_{1}^{T} X_{1}\right)^{-1}\right]\right\} \\
&= V_{1} \otimes\left(X_{1}^{T} X_{1}\right)^{-1}-\left[I_{q} \otimes\left(X_{1}^{T} X_{1}\right)^{-1} X_{1}^{T}\right]\left[Q V_{1} \otimes N_{2}\right]\left[I_{q} \otimes X_{1}\left(X_{1}^{T} X_{1}\right)^{-1}\right] \\
&+\left[I_{q} \otimes\left(X_{1}^{T} X_{1}\right)^{-1} X_{1}^{T}\right]\left[\sum_{i=0}^{\infty} Q^{i+2} V_{1} \otimes\left(P_{2} P_{1}\right)^{i} P_{2} N_{1} N_{2}\right]\left[I_{q} \otimes X_{1}\left(X_{1}^{T} X_{1}\right)^{-1}\right] \\
&+\left[I_{q} \otimes\left(X_{1}^{T} X_{1}\right)^{-1} X_{1}^{T}\right]\left[\sum_{i=0}^{\infty} Q V_{1}\left(Q^{T}\right)^{i+1} \otimes N_{2} N_{1} P_{2}\left(P_{1} P_{2}\right)^{i}\right]\left[I_{q} \otimes X_{1}\left(X_{1}^{T} X_{1}\right)^{-1}\right] \\
&+\left[I_{q} \otimes\left(X_{1}^{T} X_{1}\right)^{-1} X_{1}^{T}\right]\left[\sum_{i=0}^{\infty} Q^{i+1} \otimes\left(P_{2} P_{1}\right)^{i} P_{2} N_{1} \sum_{i=0}^{\infty} V_{1}\left(Q^{T}\right)^{i+1} \otimes N_{1} P_{2}\left(P_{1} P_{2}\right)^{i}\right]\left[I_{q} \otimes X_{1}\left(X_{1}^{T} X_{1}\right)^{-1}\right] \\
&-\left[I_{q} \otimes\left(X_{1}^{T} X_{1}\right)^{-1} X_{1}^{T}\right]\left[\sum_{i=0}^{\infty} Q^{i+1} \otimes\left(P_{2} P_{1}\right)^{i} P_{2} N_{1} \sum_{i=0}^{\infty} Q V_{1}\left(Q^{T}\right)^{i+1} \otimes N_{2} N_{1} P_{2}\left(P_{1} P_{2}\right)^{i}\right]\left[I_{q} \otimes X_{1}\left(X_{1}^{T} X_{1}\right)^{-1}\right]
\end{aligned}
$$




$$
\begin{aligned}
= & V_{1} \otimes\left(X_{1}^{T} X_{1}\right)^{-1}-\left[I_{q} \otimes\left(X_{1}^{T} X_{1}\right)^{-1} X_{1}^{T}\right]\left[Q V_{1} \otimes N_{2}\right]\left[I_{q} \otimes X_{1}\left(X_{1}^{T} X_{1}\right)^{-1}\right] \\
& +\left[I_{q} \otimes\left(X_{1}^{T} X_{1}\right)^{-1} X_{1}^{T}\right]\left[\sum_{i=0}^{\infty} Q V_{1}\left(Q^{T}\right)^{i+1} \otimes P_{2}\left(P_{1} P_{2}\right)^{i+1}-\sum_{i=0}^{\infty} Q^{i+2} V_{1} \otimes\left(P_{2} P_{1}\right)^{i+1}\right] \\
& \times\left[I_{q} \otimes X_{1}\left(X_{1}^{T} X_{1}\right)^{-1}\right] .
\end{aligned}
$$

Using $Q V_{1}=D V_{2}^{-1} D^{T}, X_{1}^{T} N_{2} X_{1}=X_{1}^{T}\left(I_{n}-P_{1} P_{2} P_{1}\right) X_{1}$, and $X_{1}^{T} P_{1}=X_{1}^{T}$, we have

$\operatorname{Cov}\left(\overline{\operatorname{Vec}}\left(B_{1}\right)\right)$

$$
\begin{aligned}
= & V_{1} \otimes\left(X_{1}^{T} X_{1}\right)^{-1}-\left[I_{q} \otimes\left(X_{1}^{T} X_{1}\right)^{-1} X_{1}^{T}\right]\left[D V_{2} D^{T} \otimes\left(I_{n}-P_{1} P_{2} P_{1}\right)\right]\left[I_{q} \otimes X_{1}\left(X_{1}^{T} X_{1}\right)^{-1}\right] \\
& -\left[I_{q} \otimes\left(X_{1}^{T} X_{1}\right)^{-1} X_{1}^{T}\right]\left\{\sum_{i=1}^{\infty} Q^{i} D V_{2}^{-1} D^{T} \otimes\left(P_{1} P_{2} P_{1}\right)^{i}-\sum_{i=1}^{\infty} D V_{2}^{-1} D^{T}\left(Q^{T}\right)^{i} \otimes\left(P_{1} P_{2} P_{1}\right)^{i+1}\right\} \\
& \times\left[I_{q} \otimes X_{1}\left(X_{1}^{T} X_{1}\right)^{-1}\right] \\
= & V_{1} \otimes\left(X_{1}^{T} X_{1}\right)^{-1}-\left[I_{q} \otimes\left(X_{1}^{T} X_{1}\right)^{-1} X_{1}^{T}\right]\left\{\sum_{i=0}^{\infty} Q^{i} D V_{2}^{-1} D^{T} \otimes\left(P_{1} P_{2} P_{1}\right)^{i} \sum_{i=0}^{\infty} D V_{2}^{-1} D^{T}\left(Q^{T}\right)^{i} \otimes\left(P_{1} P_{2} P_{1}\right)^{i+1}\right\} \\
& \times\left[I_{q} \otimes X_{1}\left(X_{1}^{T} X_{1}\right)^{-1}\right] \\
= & V_{1} \otimes\left(X_{1}^{T} X_{1}\right)^{-1}-\left[I_{q} \otimes\left(X_{1}^{T} X_{1}\right)^{-1} X_{1}^{T}\right]\left\{\sum_{i=0}^{\infty}\left[Q^{i} D V_{2}^{-1} D^{T}\right] \otimes\left[\left(P_{1} P_{2} P_{1}\right)^{i}-\left(P_{1} P_{2} P_{1}\right)^{i+1}\right]\right\} \\
& \times\left[I_{q} \otimes X_{1}\left(X_{1}^{T} X_{1}\right)^{-1}\right],
\end{aligned}
$$

where the last step uses the facts that $\left(P_{1} P_{2} P_{1}\right)^{0}=I_{n}$ and $Q^{i} D V_{2}^{-1} D^{T}=D V_{2}^{-1} D^{T}\left(Q^{T}\right)^{i}$ for $i \geq 0$.

The proof of Theorem 1 is finished.

Note that $Q^{0} D V_{2}^{-1} D^{T}=D V_{2}^{-1} D^{T} \geq 0, I_{n}-P_{1} P_{2} P_{1} \geq 0$ and for $i \geq 1$

$$
\begin{aligned}
& Q^{i} D V_{2}^{-1} D^{T}=D V_{2}^{-1} D^{T}\left(Q^{T}\right)^{i} \\
& = \begin{cases}D V_{2}^{-1} D^{T}\left(V_{1}^{-1} D V_{2}^{-1} D^{T}\right)^{k-1} V_{1}^{-1}\left(D V_{2}^{-1} D^{T} V_{1}^{-1}\right)^{k-1} D V_{2}^{-1} D^{T} \geq 0, & i=2 k-1, \\
D V_{2}^{-1} D^{T}\left(V_{1}^{-1} D V_{2}^{-1} D^{T}\right)^{k-1} V_{1}^{-1} D V_{2}^{-1} D^{T} V_{1}^{-1}\left(D V_{2}^{-1} D^{T} V_{1}^{-1}\right)^{k-1} D V_{2}^{-1} D^{T} \geq 0, & i=2 k\end{cases} \\
& \quad k=1,2, \ldots,
\end{aligned}
$$

and $\left(P_{1} P_{2} P_{1}\right)^{i}-\left(P_{1} P_{2} P_{1}\right)^{i+1} \geq 0$. Hence

$$
G=\sum_{i=0}^{\infty}\left[Q^{i} D V_{2}^{-1} D^{T}\right] \otimes\left[\left(P_{1} P_{2} P_{1}\right)^{i}-\left(P_{1} P_{2} P_{1}\right)^{i+1}\right] \geq 0 .
$$

Further, since $\operatorname{Cov}\left(\widehat{\operatorname{Vec}}\left(B_{1}\right)\right)=V_{1} \otimes\left(X_{1}^{T} X_{1}\right)^{-1}$, we have

$$
\begin{aligned}
\operatorname{Cov}\left(\overline{\operatorname{Vec}}\left(B_{1}\right)\right) & =\operatorname{Cov}\left(\widehat{\operatorname{Vec}}\left(B_{1}\right)\right)-\left[I_{q} \otimes\left(X_{1}^{T} X_{1}\right)^{-1} X_{1}^{T}\right] \cdot G \cdot\left[I_{q} \otimes X_{1}\left(X_{1}^{T} X_{1}\right)^{-1}\right] \\
& \leq \operatorname{Cov}\left(\widehat{\operatorname{Vec}}\left(B_{1}\right)\right),
\end{aligned}
$$


which means $\overline{\operatorname{Vec}}\left(B_{1}\right)$ is superior to $\widehat{\operatorname{Vec}}\left(B_{1}\right)$ in the sense of having less covariance. This result is exactly consistent with the fact that $\widehat{\operatorname{Vec}}\left(B_{1}\right)$ only uses the first regression information on $\operatorname{Vec}\left(B_{1}\right)$, whereas $\overline{\operatorname{Vec}}\left(B_{1}\right)$ combines the second regression equation with the first one via covariance adjustment.

\section{The characteristics of matrix series}

Note that for $i=1,2, \ldots$,

$$
X_{1}^{T}\left(P_{2} P_{1}\right)^{i-1} P_{2} N_{1}=0 \Longleftrightarrow X_{1}^{T}\left(P_{2} P_{1}\right)^{i-1} P_{2} N_{1} N_{2}=0 .
$$

We only need to prove that the right equality implies the left equality. Note that $X_{1}^{T}\left(P_{2} P_{1}\right)^{i-1}$ $P_{2} N_{1} N_{2}=0$ concludes $X_{1}^{T}\left(P_{2} P_{1}\right)^{i-1} N_{2} N_{1} N_{2}=0$, hence one has $X_{1}^{T}\left(P_{2} P_{1}\right)^{i-1} N_{2} N_{1} N_{2}\left(P_{1} P_{2}\right)^{i-1} X_{1}=0$, thus $X_{1}^{T}\left(P_{2} P_{1}\right)^{i-1} N_{2} N_{1}=0$, where we use $N_{1}^{2}=N_{1}$. Further, replace $N_{2}$ by $I_{n}-P_{2}$ and note that $X_{1}^{T} N_{1}=0$ and $P_{1} N_{1}=0$, we have $X_{1}^{T}\left(P_{2} P_{1}\right)^{i-1} P_{2} N_{1}=0$.

Therefore, (2.1) implies that for $i=1,2, \ldots$,

$$
\left(X_{1}^{T} X_{1}\right)^{-1} X_{1}^{T}\left(P_{2} P_{1}\right)^{i-1} P_{2} N_{1}=0 \Longleftrightarrow\left(X_{1}^{T} X_{1}\right)^{-1} X_{1}^{T}\left(P_{2} P_{1}\right)^{i-1} P_{2} N_{1} N_{2}=0,
$$

which further shows that for $i=1,2, \ldots$,

$$
Q^{i} \otimes\left(X_{1}^{T} X_{1}\right)^{-1} X_{1}^{T}\left(P_{2} P_{1}\right)^{i-1} P_{2} N_{1}=0 \Longleftrightarrow Q^{i}\left(V^{11}\right)^{-1} V^{12} \otimes\left(X_{1}^{T} X_{1}\right)^{-1} X_{1}^{T}\left(P_{2} P_{1}\right)^{i-1} P_{2} N_{1} N_{2}=0,
$$

where we note that $Q=D V_{2}^{-1} D^{T} V_{1}^{-1}$ and $Q^{i}\left(V^{11}\right)^{-1} V^{12}=-Q^{i} D V_{2}^{-1}$ and $D$ is the covariance matrix of $E_{1}$ and $E_{2}$, and that both $Q$ and $Q^{i}\left(V^{11}\right)^{-1} V^{12}$ are invertible.

Set

$$
\overline{\operatorname{Vec}}\left(B_{1}\right)_{s}=\left[I_{q} \otimes\left(X_{1}^{T} X_{1}\right)^{-1} X_{1}^{T}\right] \operatorname{Vec}\left(Y_{1}\right)+\left[\left(V^{11}\right)^{-1} V^{12} \otimes\left(X_{1}^{T} X_{1}\right)^{-1} X_{1}^{T} N_{2}\right] \operatorname{Vec}\left(Y_{2}\right) .
$$

The following theorem shows that the matrix series (1.10) only have one degeneration form $\overline{\operatorname{Vec}}\left(B_{1}\right)_{s}$.

Theorem 2. $\overline{\operatorname{Vec}}\left(B_{1}\right)_{s}$ is the unique simpler form of $\widehat{\operatorname{Vec}}\left(B_{1}\right)^{(\infty)}$.

Proof: Note that for any a fixed $i(i \geq 1)$ that: if $X_{1}^{T}\left(P_{2} P_{1}\right)^{i-1} P_{2} N_{1}=0$, then $X_{1}^{T}\left(P_{2} P_{1}\right)^{i} P_{2} N_{1}=$ $X_{1}^{T}\left(P_{2} P_{1}\right)^{i-1} P_{2}\left(I_{n}-N_{1}\right) P_{2} N_{1}=0$. Step by step, we come to

$$
X_{1}^{T}\left(P_{2} P_{1}\right)^{k-1} P_{2} N_{1}=0, \quad k=i+1, i+2, \ldots
$$

Thus, we find

$$
\begin{aligned}
& Q^{i} \otimes\left(X_{1}^{T} X_{1}\right)^{-1} X_{1}^{T}\left(P_{2} P_{1}\right)^{i-1} P_{2} N_{1}=0, \quad \text { for any a fixed } i(i \geq 1) \\
\Longrightarrow & Q^{k} \otimes\left(X_{1}^{T} X_{1}\right)^{-1} X_{1}^{T}\left(P_{2} P_{1}\right)^{k-1} P_{2} N_{1}=0, \quad k=i+1, i+2, \ldots
\end{aligned}
$$



that

On the other hand, if for any a fixed $i(i \geq 2)$ one has $X_{1}^{T}\left(P_{2} P_{1}\right)^{i-1} P_{2} N_{1}=0$, then it is easy to see

$$
\begin{aligned}
& X_{1}^{T}\left(P_{2} P_{1}\right)^{i-1}\left(I_{n}-N_{2}\right) N_{1}=0 \\
\Longrightarrow & X_{1}^{T}\left(P_{2} P_{1}\right)^{i-1} N_{2} N_{1}=0 \\
\Longrightarrow & X_{1}^{T}\left(P_{2} P_{1}\right)^{i-2} P_{2}\left(I_{n}-N_{1}\right) N_{2} N_{1}=0 \\
\Longrightarrow & X_{1}^{T}\left(P_{2} P_{1}\right)^{i-2} P_{2} N_{1} N_{2} N_{1}=0 \\
\Longrightarrow & X_{1}^{T}\left(P_{2} P_{1}\right)^{i-2} P_{2} N_{1} N_{2} N_{1} P_{2}\left(P_{1} P_{2}\right)^{i-2} X_{1}=0 \\
\Longrightarrow & X_{1}^{T}\left(P_{2} P_{1}\right)^{i-2} P_{2} N_{1} N_{2}=0 \\
\Longrightarrow & X_{1}^{T}\left(P_{2} P_{1}\right)^{i-2} P_{2} N_{1}=0,
\end{aligned}
$$

where the last step comes from the fact (2.1). Thus, step by step we conclude that

$$
\begin{aligned}
& Q^{i} \otimes\left(X_{1}^{T} X_{1}\right)^{-1} X_{1}^{T}\left(P_{2} P_{1}\right)^{i-1} P_{2} N_{1}=0, \quad \text { for any a fixed } i(i \geq 2) \\
& \Longrightarrow Q^{k} \otimes\left(X_{1}^{T} X_{1}\right)^{-1} X_{1}^{T}\left(P_{2} P_{1}\right)^{k-1} P_{2} N_{1}=0, \quad k=1,2, \ldots, i-1 .
\end{aligned}
$$

Combining (2.6) with (2.8), we know that for any a fixed $i(i \geq 1)$ if

$$
Q^{i} \otimes\left(X_{1}^{T} X_{1}\right)^{-1} X_{1}^{T}\left(P_{2} P_{1}\right)^{i-1} P_{2} N_{1}=0,
$$

then the infinite series

$$
\sum_{i=1}^{\infty}\left[Q^{i} \otimes\left(X_{1}^{T} X_{1}\right)^{-1} X_{1}^{T}\left(P_{2} P_{1}\right)^{i-1} P_{2} N_{1}\right]=0
$$

and by (2.3), concurrently we conclude that the infinite series

$$
\sum_{i=1}^{\infty}\left[Q^{i}\left(V^{11}\right)^{-1} V^{12} \otimes\left(X_{1}^{T} X_{1}\right)^{-1} X_{1}^{T}\left(P_{2} P_{1}\right)^{i-1} P_{2} N_{1} N_{2}\right]=0
$$

Hence, $\widehat{\operatorname{Vec}}\left(B_{1}\right)^{(\infty)}$ has unique simpler form $\overline{\operatorname{Vec}}\left(B_{1}\right)_{s}$ in the sense that if one term in (2.10) or (2.11) is zero, then both infinite sums turn into zero.

The proof of Theorem 2 is finished.

\section{The properties of two-stage estimator}

If the covariance matrix $V$ is unknown, then both $\widehat{\operatorname{Vec}}\left(B_{1}\right)^{(\infty)}$ and the simpler form $\overline{\operatorname{Vec}}\left(B_{1}\right)_{s}$ are not available to use. Set $\tilde{X}=\left(X_{1}, X_{2}\right)$, we estimate $V$ by

$$
\hat{V}=\frac{1}{n-R(\tilde{X})}\left(\begin{array}{c}
Y_{1}^{T} \\
Y_{2}^{T}
\end{array}\right)\left(I_{n}-P_{\tilde{X}}\right)\left(Y_{1}, Y_{2}\right)
$$

where $R(\tilde{X})$ is the $\operatorname{rank}$ of $\tilde{X}$ and $P_{\tilde{X}}=\tilde{X}\left(\tilde{X}^{T} \tilde{X}\right)^{-} \tilde{X}^{T}$. 
Following from $E\left(a^{T} A b\right)=\operatorname{trace}[A \operatorname{Cov}(b, a)]+(E a)^{T} A(E b)$ and $\left(I_{n}-P_{\tilde{X}}\right) X_{i}=0(i=1,2)$, where $a$ and $b$ denote two random vectors, we have $E\left[Y_{i}^{T}\left(I_{n}-P_{\tilde{X}}\right) Y_{i}\right]=V_{i}[n-R(\tilde{X})](i=1,2)$ and $E\left[Y_{1}^{T}\left(I_{n}-\right.\right.$ $\left.\left.P_{\tilde{X}}\right) Y_{2}\right]=D[n-R(\tilde{X})]$, which show that

$$
E \hat{V}=\left(\begin{array}{cc}
V_{1} & D \\
D^{T} & V_{2}
\end{array}\right)=V
$$

Substituting the estimator $\hat{V}$ for $V$ in the expressions of $\widehat{\operatorname{Vec}}\left(B_{1}\right)^{(\infty)}$ and $\overline{\operatorname{Vec}}\left(B_{1}\right)_{s}$, we obtain the following two two-stage estimators

$$
\widehat{\operatorname{Vec}}\left(B_{1}\right)_{2 \text {-stage }}^{(\infty)}=M(\hat{Q})\left\{\operatorname{Vec}\left(Y_{1}\right)+\left[-\hat{D} \hat{V}_{2}^{-1} \otimes N_{2}\right] \operatorname{Vec}\left(Y_{2}\right)\right\}
$$

with $M(\hat{Q})=\left[I_{q} \otimes\left(X_{1}^{T} X_{1}\right)^{-1} X_{1}^{T}\right]\left\{I_{q n}-\hat{Q} \sum_{i=0}^{\infty}\left(\hat{Q} \otimes P_{2} P_{1}\right)^{i} P_{2} N_{1}\right\}$ and $\hat{Q}=\hat{D} \hat{V}_{2}^{-1} \hat{D}^{T} \hat{V}_{1}^{-1}$, and

$$
\overline{\operatorname{Vec}}\left(B_{1}\right)_{s, 2 \text {-stage }}=\left[I_{q} \otimes\left(X_{1}^{T} X_{1}\right)^{-1} X_{1}^{T}\right] \operatorname{Vec}\left(Y_{1}\right)+\left[-\hat{D} \hat{V}_{2}^{-1} \otimes\left(X_{1}^{T} X_{1}\right)^{-1} X_{1}^{T} N_{2}\right] \operatorname{Vec}\left(Y_{2}\right)
$$

Similar to Theorem 2, we know that $\overline{\operatorname{Vec}}\left(B_{1}\right)_{s, 2 \text {-stage }}$ is the unique simpler form of $\widehat{\operatorname{Vec}}\left(B_{1}\right)_{2 \text {-stage }}^{(\infty)}$. Hence, we focus on the performances of $\overline{\operatorname{Vec}}\left(B_{1}\right)_{s, 2 \text {-stage }}$.

The matrix-variate normal distribution is a commonly used distribution in the class of matrix elliptically symmetric distributions. It plays an important role in the investigation of multivariate regression models such as the growth curve model (GCM). In what follows, in order to establish the

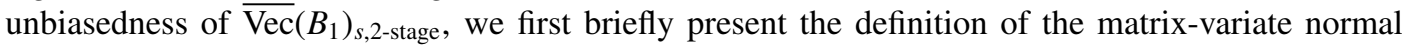
distribution as well as two related properties and then make some assumptions on the distributions of random error matrices $E_{i}(i=1,2)$.

Definition 1. A random matrix $Z$ with order $n \times q$ is said to follow a matrix-variate normal distribution if its probability function is of the form

$$
f(Z)=(2 \pi)^{-\frac{n q}{2}}[\operatorname{det}(\Sigma)]^{-\frac{q}{2}}[\operatorname{det}(\Omega)]^{-\frac{n}{2}} \exp \left(-\frac{1}{2} \operatorname{trace}\left\{\Omega^{-1}[Z-M]^{T} \Sigma^{-1}[Z-M]\right\}\right),
$$

where $M, \Sigma>0$, and $\Omega>0$ are $n \times q, n \times n$, and $q \times q$ matrices, respectively, and $\operatorname{det}(A)$ is the determinant of the square matrix $A$. In this case, it is usually denoted that $Z \sim N_{n, q}(M, \Sigma, \Omega)$.

The following two lemmas point out that the relationship between the matrix-variate and vectorvariate normal distributions and an affine transformation of a matrix-variate normal variable also follows a matrix-variate normal distribution. The readers are referred to the first chapter of Pan and Fang (2007) for more details.

Lemma 1. Let $Z$ be a $n \times q$ random matrix and $z=\operatorname{Vec}(Z)$. Then $Z \sim N_{n, q}(M, \Sigma, \Omega)$ if $z \sim N_{n q}$ $(\operatorname{Vec}(M), \Omega \otimes \Sigma)$

Lemma 2. Suppose $Z \sim N_{n, q}(M, \Sigma, \Omega)$, and that $C, A_{1}>0$, and $A_{2}>0$ are given matrices with orders $n \times q, n \times n$, and $q \times q$, respectively. Then $A_{1} Z A_{2}+C \sim N_{n, q}\left(A_{1} M A_{2}+C, A_{1} \Sigma A_{1}^{T}, A_{2} \Omega A_{2}^{T}\right)$.

In the following, we assume that in the system (1.1) the random error matrices $E_{i}(i=1,2)$ follow the matrix-variate normal distribution $N_{n, q}\left(0, I_{n}, V_{i}\right)$, which indicate that the rows of $E_{i}$ are iid random 
vectors with common distribution $N_{q}\left(0, V_{i}\right)(i=1,2)$, respectively. Thus, the rows of $E=\left(E_{1}, E_{2}\right)$ are iid random vectors with common distribution $N_{2 q}(0, V)$, i.e., $E \sim N_{n, 2 q}\left(0, I_{n}, V\right)$. Hence, by Lemmas 1 and 2 we know that

$$
\operatorname{Vec}(Y)=\operatorname{Vec}\left(Y_{1}, Y_{2}\right) \sim N_{2 n q}\left(\left[\begin{array}{cc}
I_{q} \otimes X_{1} & 0 \\
0 & I_{q} \otimes X_{2}
\end{array}\right] \operatorname{Vec}(B), V \otimes I_{n}\right)
$$

Denote $Y_{i}=\left(y_{1}^{(i)}, y_{2}^{(i)}, \ldots, y_{q}^{(i)}\right)(i=1,2)$. Then the matrix $\hat{D}=[n-R(\tilde{X})]^{-1}\left(\hat{d}_{i j}\right)_{q \times q}$ with the element

$$
\hat{d}_{i j}=\left(y_{i}^{(1)}\right)^{T}\left[I_{n}-P_{\tilde{X}}\right] y_{j}^{(2)}=(\operatorname{Vec}(Y))^{T}\left[O_{i, q+j}(2 q \times 2 q) \otimes\left(I_{n}-P_{\tilde{X}}\right)\right] \operatorname{Vec}(Y),
$$

where the matrix $O_{i, q+j}(2 q \times 2 q)$ with order $2 q \times 2 q$ consists of all zeros only except the element in the $i^{\text {th }}$ row and the $(q+j)^{\text {th }}$ column is one. Similarly, the $(i, j)^{\text {th }}$ element of $\hat{V}_{2}$ is equal to

$$
(\operatorname{Vec}(Y))^{T}\left[O_{q+i, q+j}(2 q \times 2 q) \otimes\left(I_{n}-P_{\tilde{X}}\right)\right] \operatorname{Vec}(Y)
$$

where the $2 q \times 2 q$ order matrix $O_{q+i, q+j}(2 q \times 2 q)$ consists of all zeros only; except the element in the $(q+i)^{t h}$ row and the $(q+j)^{\text {th }}$ column is one.

Note that $\left[I_{q} \otimes\left(X_{1}^{T} X_{1}\right)^{-1} X_{1}^{T} N_{2}\right] \operatorname{Vec}\left(Y_{2}\right)=\left[\mathbf{0}_{q p_{1} \times n q}, I_{q} \otimes\left(X_{1}^{T} X_{1}\right)^{-1} X_{1}^{T} N_{2}\right] \operatorname{Vec}(Y)$. Hence, using $X_{1}^{T} N_{2}\left[I_{n}-P_{\tilde{X}}\right]=0$ and following from the discriminant condition of independence of the linear function and quadratic function of normal variables and the following easily verified facts:

$$
\left[\mathbf{0}_{q p_{1} \times n q}, I_{q} \otimes\left(X_{1}^{T} X_{1}\right)^{-1} X_{1}^{T} N_{2}\right]\left[V \otimes I_{n}\right]\left[O_{i, q+j}(2 q \times 2 q) \otimes\left(I_{n}-P_{\tilde{X}}\right)\right]=0,
$$

and

$$
\left[\mathbf{0}_{q p_{1} \times n q}, I_{q} \otimes\left(X_{1}^{T} X_{1}\right)^{-1} X_{1}^{T} N_{2}\right]\left[V \otimes I_{n}\right]\left[O_{q+i, q+j}(2 q \times 2 q) \otimes\left(I_{n}-P_{\tilde{X}}\right)\right]=0 .
$$

We know that

$$
\begin{aligned}
E\left[\overline{\operatorname{Vec}}\left(B_{1}\right)_{s, 2-\text { stage }}\right]= & {\left[I_{q} \otimes\left(X_{1}^{T} X_{1}\right)^{-1} X_{1}^{T}\right]\left(I_{q} \otimes X_{1}\right) \operatorname{Vec}\left(B_{1}\right) } \\
& +\left[\left(E\left(-\hat{D} \hat{V}_{2}^{-1}\right)\right) \otimes\left(X_{1}^{T} X_{1}\right)^{-1} X_{1}^{T} N_{2}\right]\left(I_{q} \otimes X_{2}\right) \operatorname{Vec}\left(B_{2}\right) \\
= & \operatorname{Vec}\left(B_{1}\right) .
\end{aligned}
$$

Thus, we obtain the following theorem, which states the unbiasedness of the two-stage estimator.

Theorem 3. Under the assumptions that $E_{i} \sim N_{n, q}\left(0, I_{n}, V_{i}\right)(i=1,2)$, the two-stage estimator $\overline{\operatorname{Vec}}\left(B_{1}\right)_{s, 2 \text {-stage }}$ is unbiased, i.e., $E\left[\overline{\operatorname{Vec}}\left(B_{1}\right)_{s, 2 \text {-stage }}\right]=\operatorname{Vec}\left(B_{1}\right)$.

In the following, we refer to Grunfeld's data in Maddala (1977) and present two simulation studies to compare the performances of $\overline{\mathrm{Vec}}\left(B_{1}\right)_{s, 2 \text {-stage }}$ with those of $\widehat{\operatorname{Vec}}\left(B_{1}\right)$ under the conditions that there are some known relationships between the design matrices $X_{1}$ and $X_{2}$ and no relationships between $X_{1}$ and $X_{2}$, respectively. 
Table 1: Comparisons between the two-stage estimator and the least square estimator

\begin{tabular}{ccccc}
\hline \hline$\rho$ & $n$ & $\operatorname{trace}\left(S\left(\hat{B}_{1}\right)\right)$ & $\operatorname{trace}\left(S\left(\bar{B}_{1, s, 2 \text {-stage }}\right)\right)$ & trace $\left(S\left(\bar{B}_{1, s}\right)\right)$ \\
\hline \multirow{2}{*}{0.2} & 10 & 12.7118 & 12.8775 & 12.7718 \\
& 20 & 30.0456 & 30.2155 & 30.0873 \\
& 50 & 141.1215 & 141.9674 & 141.3323 \\
\hline \multirow{2}{*}{0.5} & 10 & 15.3561 & 15.4368 & 15.5200 \\
& 20 & 35.3589 & 35.3747 & 35.3775 \\
& 50 & 108.2523 & 108.2895 & 108.2894 \\
\hline \multirow{2}{*}{0.7} & 10 & 7.7018 & 8.0020 & 7.9504 \\
& 20 & 42.4375 & 45.8153 & 104.2045 \\
\hline & 50 & 103.9155 & 104.3501 & 18.6689 \\
& 10 & 18.6055 & 18.6758 & 37.2077 \\
& 20 & 35.6630 & 37.5563 & 122.2700 \\
\hline \hline
\end{tabular}

(I) The case that $X_{1}=\left(X_{2}, L\right)$

Where the system (1.1) is of the form $Y_{i}=X_{i} B_{i}+E_{i}(i=1,2)$ with $E=\left(E_{1}, E_{2}\right) \sim N_{n, 4}\left(0, I_{n}, V\right)$, and

$$
B_{1}=\left(\begin{array}{ll}
1 & 1 \\
1 & 2 \\
1 & 3
\end{array}\right), \quad B_{2}=\left(\begin{array}{cc}
1 & 6 \\
-3 & 2
\end{array}\right), \quad V=\left(\begin{array}{llll}
1 & 0 & \rho & 0 \\
0 & 1 & 0 & \rho \\
\rho & 0 & 1 & 0 \\
0 & \rho & 0 & 1
\end{array}\right)
$$

Set $S\left(B_{1}\right)=\left(Y_{1}-X_{1} B_{1}\right)^{T}\left(Y_{1}-X_{1} B_{1}\right)$. Note that the estimator $\hat{B}_{1}=\left(X_{1}^{T} X_{1}\right)^{-1} X_{1}^{T} Y_{1}$ given by (1.3), which corresponds to the LSE $\widehat{\operatorname{Vec}}\left(B_{1}\right)$, actually makes the residual sum of squares (in the sense of nonnegative definite), trace of $S\left(B_{1}\right)$, determinant of $S\left(B_{1}\right)$ and the largest eigenvalue of $S\left(B_{1}\right)$ achieve their minimums (Muirhead, 1982). Therefore, under the four different criteria of measurement, if only the first equation $Y_{1}=X_{1} B_{1}+E_{1}$ is used then the LSE of the regression coefficient $B_{1}$ are completely identical (Fang and Zhang, 1990). Thus, without loss of generality, we illustrate the superiorities of $\overline{\operatorname{Vec}}\left(B_{1}\right)_{s, 2 \text {-stage }}$ by comparing $\operatorname{trace}\left(S\left(\hat{B}_{1}\right)\right)$ with trace $\left(S\left(\bar{B}_{1, s, 2 \text {-stage }}\right)\right)$, where $\bar{B}_{1, s, 2 \text {-stage }}=$ $\left(X_{1}^{T} X_{1}\right)^{-1} X_{1}^{T} Y_{1}+\left(X_{1}^{T} X_{1}\right)^{-1} X_{1}^{T} N_{2} Y_{2}\left(-\hat{D} \hat{V}_{2}^{-1}\right)^{T}$, which corresponds to $\overline{\operatorname{Vec}}\left(B_{1}\right)_{s, 2-\text { stage }}$. We also present the values of trace $\left(S\left(\bar{B}_{1, s}\right)\right)$ for contrast, where $\bar{B}_{1, s}=\left(X_{1}^{T} X_{1}\right)^{-1} X_{1}^{T} Y_{1}+\left(X_{1}^{T} X_{1}\right)^{-1} X_{1}^{T} N_{2} Y_{2}\left(-D V_{2}^{-1}\right)^{T}$ corresponds to (2.4).

In Table 1, based on different combinations of the correlation $\rho$ and sample size, we present some numerical demonstrations to compare $\operatorname{trace}\left(S\left(\bar{B}_{1, s, 2 \text {-stage }}\right)\right)$ with trace $\left(S\left(\hat{B}_{1}\right)\right)$ and $\operatorname{trace}\left(S\left(\bar{B}_{1, s}\right)\right)$, which

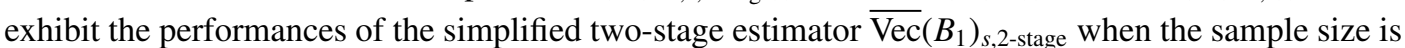
relatively small and moderate. Consequently, we find that the performance of the two-stage estimator tends to improve as the sample size increases. However, it also depends on the correlation $\rho$, and especially when $n \geq 20$ and $\rho \geq 0.5$, we easily see that $\left|\operatorname{trace}\left(S\left(\bar{B}_{1, s, 2 \text {-stage }}\right)\right)-\operatorname{trace}\left(S\left(\bar{B}_{1, s}\right)\right)\right|<$ $\left|\operatorname{trace}\left(S\left(\hat{B}_{1}\right)\right)-\operatorname{trace}\left(S\left(\bar{B}_{1, s}\right)\right)\right|$, which shows that the two-stage estimator $\overline{\operatorname{Vec}}\left(B_{1}\right)_{s, 2 \text {-stage }}$ is closer to $\overline{\operatorname{Vec}}\left(B_{1}\right)_{s}$.

(II) The case that there are no relationships between $X_{1}$ and $X_{2}$

In this case we assume that the system (1.1) has the same form as (3.10) but there are no relationships between $X_{1}$ and $X_{2}$. The simulations are presented below. From Table 2, we see that $\operatorname{trace}\left(S\left(\bar{B}_{1, s, 2 \text {-stage }}\right)\right)$ is getting closer to trace $\left(S\left(\bar{B}_{1, s}\right)\right)$, which implies that the two-stage estimator $\overline{\mathrm{Vec}}\left(B_{1}\right)_{s, 2 \text {-stage }}$ is becoming better than the LSE $\widehat{\operatorname{Vec}}\left(B_{1}\right)$ as the sample size goes large $(n \geq 20$ or 
Table 2: Comparisons between the two-stage estimator and the least square estimator

\begin{tabular}{ccccc}
\hline \hline$\rho$ & $n$ & $\operatorname{trace}\left(S\left(\hat{B}_{1}\right)\right)$ & $\operatorname{trace}\left(S\left(\bar{B}_{1, s, 2-\text { stage }}\right)\right)$ & $\operatorname{trace}\left(S\left(\bar{B}_{1, s}\right)\right)$ \\
\hline \multirow{2}{*}{0.2} & 10 & 16.4822 & 16.7363 & 16.5106 \\
& 20 & 45.1567 & 45.3013 & 45.1656 \\
& 50 & 90.6848 & 90.7475 & 91.0296 \\
\hline \multirow{2}{*}{0.5} & 10 & 11.7975 & 12.4334 & 12.0509 \\
& 20 & 30.6931 & 31.4717 & 31.2451 \\
& 50 & 91.1112 & 91.1782 & 15.2101 \\
\hline \multirow{2}{*}{0.7} & 10 & 15.0877 & 15.4275 & 34.6478 \\
& 20 & 34.0316 & 34.9262 & 79.4065 \\
\hline \multirow{2}{*}{0.9} & 50 & 78.8424 & 79.1979 & 9.0277 \\
& 10 & 7.7416 & 8.8334 & 38.6530 \\
& 20 & 37.1639 & 39.6293 & 106.7741 \\
\hline \hline
\end{tabular}

larger), also the fact depends on the value of the correlation $\rho(\geq 0.5)$. This is because that from the viewpoint of covariance adjustment the one-step covariance adjustment estimator $\widehat{\operatorname{Vec}}\left(B_{1}\right)^{(1)}$, which is exactly equal to $\overline{\operatorname{Vec}}\left(B_{1}\right)_{s}$, is superior to $\widehat{\operatorname{Vec}}\left(B_{1}\right)$ in the sense of having less covariance even though there are no relationships between $X_{1}$ and $X_{2}$. Hence, the simulation study discloses the tendency of $\overline{\operatorname{Vec}}\left(B_{1}\right)_{s, 2 \text {-stage }}$ performing better, which is consistent with a two-stage estimator that incorporates more information.

\section{An illustrating example}

The GCM is a generalized multivariate analysis-of-variance model, which is useful especially for investigating growth problems on short time series in economics, biology and medical research (see Lee and Geisser 1972, Pan and Fang 2007). The seemingly unrelated GCMs are defined as

$$
\left\{\begin{array}{l}
Y_{1}=X_{1} B_{1} Z_{1}+E_{1} \\
Y_{2}=X_{2} B_{2} Z_{2}+E_{2}
\end{array}\right.
$$

where $Y_{i}$ are $n \times q$ observation matrices, $X_{i}$ and $Z_{i}$ are known design matrices of full column rank and full row rank, respectively, and the regression parameters $B_{1}$ and $B_{2}$ are unknown. The assumptions on $E_{1}$ and $E_{2}$ are the same as those in the system (1.1).

Therefore, without considering the interactions between the two equations, we obtain the LSE of $B_{1}$ from the first equation as

$$
\hat{B}_{1}=\left(X_{1}^{T} X_{1}\right)^{-1} X_{1}^{T} Y_{1} V_{1}^{-1} Z_{1}^{T}\left(Z_{1} V_{1}^{-1} Z_{1}^{T}\right)^{-1}
$$

which is unbiased and the corresponding covariance $\operatorname{Cov}\left(\hat{B}_{1}\right)=\operatorname{Cov}\left(\operatorname{Vec}\left(\hat{B}_{1}\right)\right)=\left(Z_{1} V_{1}^{-1} Z_{1}^{T}\right)^{-1} \otimes$ $\left(X_{1}^{T} X_{1}\right)^{-1}$. However, combining the information of the second equation and the assumption $X_{1}^{T} X_{2}=0$, we obtain the system LSE for $B_{1}$ as

$$
\bar{B}_{1}=\left(X_{1}^{T} X_{1}\right)^{-1} X_{1}^{T}\left(Y_{1} V^{11}+Y_{2} V^{21}\right) Z_{1}^{T}\left(Z_{1} V^{11} Z_{1}^{T}\right)^{-1}
$$

which is unbiased and with less covariance

$$
\operatorname{Cov}\left(\bar{B}_{1}\right)=\operatorname{Cov}\left(\operatorname{Vec}\left(\bar{B}_{1}\right)\right)=\left(Z_{1} V^{11} Z_{1}^{T}\right)^{-1} \otimes\left(X_{1}^{T} X_{1}\right)^{-1},
$$

which is less than $\operatorname{Cov}\left(\hat{B}_{1}\right)$ since $V_{1}^{-1} \leq\left(V_{1}-D V_{2}^{-1} D^{T}\right)^{-1}=V^{11}$ and correspondingly $\left(Z_{1} V_{1}^{-1} Z_{1}^{T}\right)^{-1} \geq$ $\left(Z_{1} V^{11} Z_{1}^{T}\right)^{-1}$. 
Table 3: Comparisons between several estimators under the matrix 2 norm

\begin{tabular}{ccccc}
\hline \hline$\rho$ & $n$ & norm $\left(\hat{B}_{1}-B_{1}\right)$ & norm $\left(\bar{B}_{1,2 \text {-stage }}-B_{1}\right)$ & norm $\left(\bar{B}_{1}-B_{1}\right)$ \\
\hline \multirow{2}{*}{0.2} & 10 & 0.1931 & 0.2668 & 0.1879 \\
& 20 & 0.0183 & 0.0193 & 0.0182 \\
& 50 & 0.2259 & 0.2266 & 0.2205 \\
\hline \multirow{2}{*}{0.5} & 10 & 0.2013 & 0.2283 & 0.1679 \\
& 20 & 0.0186 & 0.0177 & 0.0171 \\
& 50 & 0.2557 & 0.2271 & 0.2198 \\
\hline \multirow{3}{*}{0.7} & 10 & 0.1896 & 0.1872 & 0.1383 \\
& 20 & 0.0184 & 0.0134 & 0.0127 \\
& 50 & 0.2532 & 0.1820 & 0.1763 \\
\hline \multirow{2}{*}{0.9} & 10 & 0.1923 & 0.1138 & 0.0847 \\
& 20 & 0.0173 & 0.0085 & 0.0079 \\
& 50 & 0.2471 & 0.1133 & 0.1103 \\
\hline \hline
\end{tabular}

In the case that the covariance matrix $V$ is unknown, under the assumption that $E=\left(E_{1}, E_{2}\right) \sim$ $N_{n, 2 q}\left(0, I_{n}, V\right)$, we use the same form estimator as that of the equation (3.1) to estimate $V$, which is easily shown to be unbiased. Hence, a two-stage estimator for $B_{1}$ is defined as

$$
\bar{B}_{1,2 \text {-stage }}=\left(X_{1}^{T} X_{1}\right)^{-1} X_{1}^{T}\left(Y_{1} \hat{V}^{11}+Y_{2} \hat{V}^{21}\right) Z_{1}^{T}\left(Z_{1} \hat{V}^{11} Z_{1}^{T}\right)^{-1},
$$

where $\hat{V}^{11}=\left(\hat{V}_{1}-\hat{D} \hat{V}_{2}^{-1} \hat{D}^{T}\right)^{-1}$ and $\hat{V}^{21}=-\hat{V}_{2}^{-1} \hat{D}^{T} \hat{V}^{11}$. Analogous to the previous discussions, we can establish the unbiasedness of the estimator $\bar{B}_{1,2 \text {-stage }}$.

In the following, we illustrate a simulation study to compare the performances of $\bar{B}_{1,2 \text {-stage }}$ with those of $\hat{B}_{1}$ under the matrix 2-norm criterion, where the 2-norm of a matrix $A$ is given by $\|A\|_{2}=$ $\|\operatorname{Vec}(A)\|_{2}=\left(\sum_{i} \sum_{j} a_{i j}^{2}\right)^{1 / 2}$. The performances of $\bar{B}_{1}$ are also presented as a contrast. In each simulation, a sample of size $n$ observations is randomly generated from a $2 q$-variate normal distribution with mean zero and covariance matrix $V$, which is considered as the error matrix $E_{n \times 2 q}=\left(E_{1}, E_{2}\right)$. Next, $\hat{B}_{1}, \bar{B}_{1,2 \text {-stage }}$, and $\bar{B}_{1}$ are calculated in each simulation. Simulations are repeated 500 times and the matrix 2-norms of the average values of $\hat{B}_{1}-B_{1}, \bar{B}_{1,2 \text {-stage }}-B_{1}$, and $\bar{B}_{1}-B_{1}$ are given in Table 3 .

Three cases are studied. The first of them corresponds to $n=10$, the second one considers the case of $n=20$ and the third one corresponds to the case of $n=50$. All cases adopt the same $V$ as (3.10), but with the correlation $\rho$ having a number of alternative values.

Simulations for the case (i) with

$$
\begin{aligned}
X_{1}^{T} & =\left(\begin{array}{cccccccccc}
616 & -302 & 1 & 9 & 23 & 19 & 25 & 26 & 17 & 11 \\
614 & -302 & 27 & 15 & 20 & 26 & 6 & 24 & 25 & 8 \\
37 & -28 & 1 & 2 & 5 & -4 & -2 & 9 & 1 & 4
\end{array}\right), \\
X_{2}^{T} & =\left(\begin{array}{llllllllll}
1 & 4 & 2 & 5 & 6 & 3 & 5 & 2 & 5 & 8 \\
2 & 6 & 3 & 4 & 2 & 1 & 6 & 8 & 5 & 3
\end{array}\right), \\
B_{1} & =\left(\begin{array}{ll}
1 & 1 \\
1 & 2 \\
1 & 3
\end{array}\right), \quad B_{2}=\left(\begin{array}{cc}
1 & 6 \\
-3 & 2
\end{array}\right), \quad Z_{1}=\left(\begin{array}{ll}
1 & 2 \\
3 & 4
\end{array}\right), \quad Z_{2}=\left(\begin{array}{cc}
1 & 5 \\
6 & 0.5
\end{array}\right) .
\end{aligned}
$$

Simulations for the case (ii) with $X_{1}^{T}$ being

$$
\left(\begin{array}{cccccccccccccccccccc}
-948 & 15 & -2654 & 5 & 25 & 35 & 10 & 30 & 45 & 0 & 20 & 5 & 15 & 70 & 65 & 75 & 60 & 90 & 60 & 20 \\
-1069 & 30 & -3407 & 15 & 80 & 55 & 35 & 85 & 70 & 40 & 25 & 95 & 75 & 5 & 10 & 20 & 90 & 65 & 45 & 100 \\
-1766 & 60 & -2713 & 100 & 10 & 70 & 75 & 50 & 65 & 90 & 40 & 45 & 5 & 25 & 85 & 55 & 35 & 30 & 80 & 15
\end{array}\right)
$$


and

$$
X_{2}^{T}=\left(\begin{array}{cccccccccccccccccccc}
1 & 2 & 3 & 4 & 5 & 6 & 7 & 8 & 9 & 10 & 11 & 12 & 13 & 14 & 15 & 16 & 17 & 18 & 19 & 20 \\
2 & 4 & 1 & 6 & 8 & 7 & 12 & 4 & 8 & 4 & 18 & 5 & 3 & 9 & 4 & 7 & 3 & 11 & 6 & 2
\end{array}\right),
$$

where $B_{1}, B_{2}, Z_{1}$, and $Z_{2}$ are the same as the case (i).

Simulations for the case (iii) with $X_{1}=\left[a_{1}, a_{2}, a_{3}\right]_{50 \times 3}$ being randomly generated and $X_{2}=$ $\left[a_{4}, a_{5}\right]_{50 \times 2}$ being obtained from the null space of $X_{1}^{T}$, and in this case $B_{1}, B_{2}, Z_{1}$, and $Z_{2}$ remain the same as those of the case (i).

From Table 3, except the situations that $\rho=0.2$ and $\rho=0.5$ with $n=10$, we find that norm $\left(\bar{B}_{1,2 \text {-stage }}\right.$ $\left.-B_{1}\right)$ is uniformly smaller than norm $\left(\hat{B}_{1}-B_{1}\right)$, which shows that the two-stage estimator $\bar{B}_{1,2 \text {-stage }}$ is closer to the true value $B_{1}$ than the LSE $\hat{B}_{1}$.

\section{Concluding remarks}

In summary, we have investigated regression coefficients estimation and inference for the system of two multivariate SURs. Note that we focus on the estimation problem of $B_{1}$ since the positions of $B_{1}$ and $B_{2}$ are equipotent. In Section 1, we find that together with another equation information the estimator of regression coefficients can be presented as a matrix power series via the method of covariance adjustment. In Section 2, we further indicate that the matrix series has exactly one simpler form which is just the one-step covariance adjustment estimator of the regression coefficients. In Section 3 , in the case that the covariance matrix of the system is unknown, we illustrate that the degeneration form of the two-stage estimator sequence is unique, and an unbiased two-stage estimator is proposed and numerical simulations are also presented to verify its superiority. The results established in the present paper enrich the existing results since they include Zellner's univariate SURs as a special case.

\section{Acknowledgements}

Partly supported by NNSF of China (11371051).

\section{References}

Alkhamisi MA (2010). Simulation study of new estimators combining the SUR ridge regression and the restricted least squares methodologies, Statistical Papers, 51, 651-672.

Baksalary JK (1991). Covariance adjustment in biased estimation, Computational Statistics \& Data Analysis, 12, 221-230.

Fang KT and Zhang YT (1990). Generalized Multivariate Analysis, Springer-Verlag and Science Press, Berlin and Beijing.

Gupta AK and Kabe DG (1998). A note on a result for two SUR models, Statistical Papers, 39, $417-421$.

Lee JC and Geisser S (1972). Growth curve prediction, Sankhya A, 34, 393-412.

Liu AY (2002). Efficient estimation of two seemingly unrelated regression equations, Journal of Multivariate Analysis, 82, 445-456.

Liu JS (2000). MSEM dominance of estimators in two seemingly unrelated regressions, Journal of Statistical Planning and Inference, 88, 255-266.

Maddala GS (1977). Econometrics, McGraw-Hill, New York.

Muirhead RJ (1982). Aspects of Multivariate Statistical Theory, Wiley and Sons, New York. 
Pan JX and Fang KT (2007). Growth Curve Models and Statistical Diagnostics, Science Press, Beijing.

Percy DF (1992). Prediction for seemingly unrelated regressions, Journal of the Royal Statistical Society Series B (Methodological), 54, 243-252.

Rao CR (1967). Least square theory using an estimated dispersion matrix and its application to measurement of signal. In Proceedings of the Fifth Berkeley Symposium on Mathematical Statistics and Probability, LeCam, L. M. \& Neyman, J. (eds), 1, 355-372.

Revankar NS (1974). Some finite sample results in the context of two seemingly unrelated regression equations, Journal of the American Statistical Association, 69, 187-190.

Schmidt P (1977). Estimation of seemingly unrelated regressions with unequal numbers of observations, Journal of Econometrics, 5, 365-377.

Shukur G and Zeebari Z (2012). Median regression for SUR models with the same explanatory variables in each equation, Journal of Applied Statistics, 39, 1765-1779.

Srivastava VK and Giles DEA (1987). Seemingly Unrelated Regression Equations Models, Marcel Dekker, New York.

Velu R and Richards J (2008). Seemingly unrelated reduced-rank regression model, Journal of Statistical Planning and Inference, 138, 2837-2846.

Wang SG (1989). A new estimate of regression coefficients in seemingly unrelated regression system, Science in China Series A, 32, 808-816.

Zellner A (1962). An efficient method of estimating seemingly unrelated regressions and tests for aggregation bias, Journal of the American Statistical Association, 57, 348-368.

Zellner A (1963). Estimators of seemingly unrelated regression equations: some exact finite sample results, Journal of the American Statistical Association, 58, 977-992.

Zhou B, Xu Q, and You J (2011). Efficient estimation for error component seemingly unrelated nonparametric regression models, Metrika, 73, 121-138. 